\title{
Pd@magnetic Carbon Dot Immobilized on the Cyclodextrin Nanosponges - Biochar Hybrid as an Efficient Hydrogenation Catalyst
}

\author{
Samahe Sadjadi ${ }^{1}$, Majid M. Heravi ${ }^{2}$, Leila Mohammadi ${ }^{2}$, Masoumeh Malmir ${ }^{2}$
}

1- Gas Conversion Department, Faculty of Petrochemicals, Iran Polymer and Petrochemicals Institute, PO Box 14975-112, Tehran, Iran

2- Department of Chemistry, School of Science, Alzahra University, PO Box 1993891176,

Vanak, Tehran, Iran

\begin{abstract}
With the aim of benefiting from the advantage of magnetic catalysts, chemistry of cyclodextrin, i. e. the capability of formation of inclusion complex and acting as phase transferring agent, as well as biochars as biocompatible, cost-effective and efficient catalyst support, for the first time a ternary hybrid system composed of cyclodextrin nanosponges (CDNS), palladated magnetic carbon quantum dot (Pd@CQDs@Fe) and Bell-pepper-derived biochar (Biochar) was designed and prepared through functionalization of CDNS and Biochar and their covalent conjugation followed by immobilization of Pd@CQDs@Fe. The hybrid system was characterized and then applied as a magnetic heterogeneous catalyst for promoting hydrogenation of nitroarenes. The results confirmed high catalytic activity, selectivity and recyclability of the catalyst (up to ten reaction runs) as well as low Pd leaching. Comparing the catalytic activity of the catalyst with some control samples, the contribution of CDNS, Biochar and CQDs@Fe to the catalysis was confirmed.
\end{abstract}

Keywords: Biochar, Carbon quantum dots, Cyclodextrin nanosponges, Hydrogenation, Magnetic catalyst 